\title{
INNOVACIÓN EN DOCENCIA DE MINERALOGÍA A PARTIR DE LA REALIZACIÓN DE VÍDEOS SOBRE CONTENIDOS PRÁCTICOS
}

Teaching innovation from the production of videos on practices of mineralogy Inovação no ensino de mineralogia através da produção de vídeos sobre trabalhos práticos

Financiación del trabajo: Proyecto “Diseño de experiencias prácticas de enseñanzaaprendizaje en relación con la musealización virtual de la Colección Geológica de la Universidad de León", Plan de Apoyo a la Innovación Docente de la Universidad de León (PAID 2016).

\section{Fernando Gómez-Fernández (1) \\ María Fernández Raga (2) \\ Héctor Alaiz Moretón (3) \\ Gabriel Búrdalo Salcedo (4)}

(1) School of Mining Engineering. University of León, Spain. Telephone: +34 987293528. E-mail: f.gomez@unileon.es

(2) Department of Applied Chemistry and Physics. University of Leon, Spain. E-mail: maria.raga@unileon.es

(3) Department of Electrical Systems Engineering and Automation. University of Leon, Spain. hector.moreton@unileon.es

(4) Department of Applied Chemistry and Physics. University of Leon, Spain. E-mail: gabriel.burdalo@unileon.es

\section{Resumen}

En la página web http://laboratorio.wesped.es/, se muestran vídeos de ensayos de propiedades físicas de minerales, realizados por alumnos como una práctica de la asignatura “Mineralogía y Petrografía” (Grado en Ingeniería Minera), en el marco de un proyecto de innovación docente. Los minerales utilizados en la experiencia pertenecen a la Colección Geológica de la Universidad de León, formada por más de 2000 ejemplares. La experiencia fue evaluada por dos vías: a) comparación de los resultados académicos de los alumnos en esta práctica con resultados equivalentes de cursos Innovación en docencia de mineralogía a partir de la realización de vídeos sobre contenidos prácticos 
académicos anteriores y, b) realización de una encuesta de satisfacción. La evaluación de la experiencia, aunque muy satisfactoria, no es concluyente, dado el reducido número de alumnos participantes. Por ello, esta experiencia se prolongará en el tiempo y se ampliará a otras asignaturas de los Grados de Ingeniería Minera y de la Energía. Palabras clave: Innovación docente; Mineralogía; Museo virtual

\begin{abstract}
At http://laboratorio.wesped.es/, videos of tests of physical properties of minerals are shown. This videos were made by students as a practice of the subject "Mineralogy and Petrography" (Degree in Mining Engineering) in the framework of a teacher innovation project. The minerals used in the experience belong to the Geological Collection of the University of León, formed by more than 2000 specimens. This activity was evaluated in two ways: a) comparing the academic results of the students in this practice with equivalent results of previous academic courses and, b) conducting a satisfaction survey. The evaluation of the experience, although was very satisfactory, is not conclusive, given the small number of students participants. Therefore, the project will be extended in time and will be extended to other subjects of the Degrees in Mining and Energy Engineering.
\end{abstract}

Keywords: Teaching innovation; Mineralogy; Virtual Museum

\title{
Resumo
}

Em http://laboratorio.wesped.es/, se mostram vídeos de testes de propriedades físicas dos minerais, feitas pelos estudantes como uma prática da matéria "Mineralogia e Petrografia" (Licenciatura em Engenharia de Minas), no âmbito de um projeto docente. Minerais utilizados na experiência pertencem à Coleção Geológica da Universidade de León, que consiste de mais de 2000 cópias. O projeto foi avaliado de duas maneiras: a) comparando o desempenho acadêmico dos alunos na prática, com resultados equivalentes de anos lectivos anteriores e, b) realização de uma pesquisa de satisfação. A avaliação da experiência, embora muito satisfatório, não é conclusivo, dado o pequeno número de alunos participantes. Portanto, o projeto docente será estendido no tempo e será estendido a outros assuntos nos Licenciaturas em Engenharia de Minas e de Energia.

Palavras-chave: Inovação na prática docente; Mineralogia; Museu Virtual 


\section{Introducción}

La Colección Geológica de la Universidad de León, con 2000 ejemplares de minerales, rocas y fósiles, ofrece un marco de innovación docente en relación con asignaturas de contenido geológico que se imparten en los Grados de Ingeniería Minera e Ingeniería de la Energía.

La innovación docente realizada estuvo ligada al proyecto de la Universidad de León de título "Diseño de experiencias prácticas de enseñanza-aprendizaje en relación con la musealización virtual de la Colección Geológica de la Universidad de León” (PAID2016). Los antecedentes más próximos a esta innovación los encontramos en Sabbatini (2004), Alfonso et al. (2009) y Fernández Caliani et al. (2012).

\section{Contextualización}

La experiencia educativa se realizó durante el curso académico 2016-17, en el marco de la práctica de "Reconocimiento y clasificación de minerales" de la asignatura “Mineralogía y Petrografía”, de tercer curso del Grado en Ingeniería Minera de la Universidad de León. En la experiencia docente participaron diez alumnos, que se corresponden con los alumnos pendientes de evaluación positiva en estos contenidos prácticos de la asignatura.

\section{Diseño y desarrollo}

Los alumnos, en grupos de dos y bajo la supervisión del profesor de la asignatura, elaboraron guiones y realizaron vídeos con sus teléfonos móviles sobre algunas propiedades físico-químicas relevantes para la identificación y clasificación de minerales. Las propiedades ensayadas fueron: color, raya, magnetismo, exfoliación, dureza, birrefringencia, fluorescencia y reacción al $\mathrm{HCl}$ (10\%) en frío. Posteriormente, los vídeos fueron evaluados por el conjunto de alumnos y el profesor. En esta evaluación, por una parte, se tuvieron en cuenta criterios científicos y didácticos (contenido científico, propiedad y estructuración de contenidos, etc.), así como otros criterios, relacionados con la calidad técnica del vídeo (luz, sonido, ritmo, duración, encuadre de la imagen, etc.). Aquellos videos considerados mejores están disponibles en la plataforma Youtube a través del link "Videos de Ensayos", creado en la web del Museo Mineralógico Virtual de la Universidad de León (http://laboratorio.wesped.es/). 


\section{Evaluación y conclusiones}

La evaluación de la experiencia se realizó por dos vías: a) Encuesta de satisfacción entre alumnos y, b) Comparación del promedio de las calificaciones obtenidas en la práctica de "Reconocimiento y clasificación de minerales", tras la actividad de innovación realizada, con la media de las calificaciones obtenidas por alumnos en los tres cursos académicos anteriores.

La encuesta de satisfacción constó de diez ítems, sin que hubiera respuestas obligatorias. A los alumnos se les preguntó si les había resultado útil y sencilla la práctica de la grabación en vídeo de los ensayos de propiedades físicas de los minerales y la elaboración de los guiones de los vídeos (Figura 1). El 90\% respondió afirmativamente. En la Figura 2, se puede observar cómo al 70\% de los alumnos le han sido suficientes las indicaciones y explicaciones previas, dadas por el profesor.

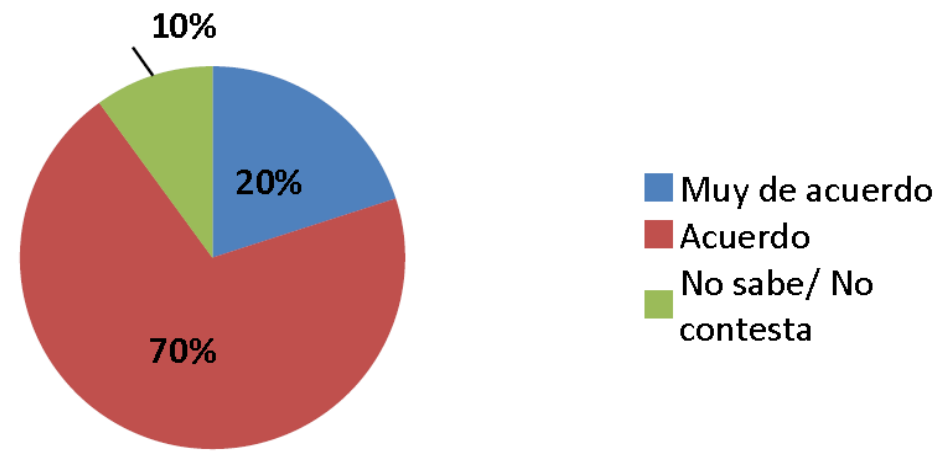

Figura 1.

Respuestas de los alumnos a las preguntas: "La práctica de grabación en vídeo de los ensayos de propiedades físicas de minerales me ha resultado útil y sencilla" y "La elaboración de los guiones de los vídeos realizados me ha resultado útil y sencilla".

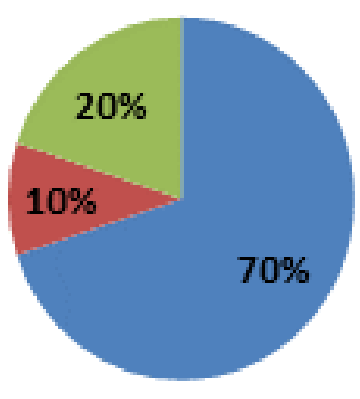

Muy de acuerdo

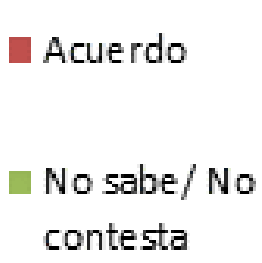

Figura 2.

Respuestas a la pregunta: ¿Crees que hubieras necesitado más explicaciones previas a la realización de las prácticas? 
La publicación de los mejores vídeos en la plataforma del Museo Geológico Virtual de la Universidad de León, no resultó una motivación para los encuestados, a la hora de realizarlos. Sin embargo, el 80\% de los alumnos piensa que ha aprendido más realizando esta práctica que utilizando métodos convencionales (Figura 3), y considera que la metodología desarrollada es adecuada para el desarrollo de esta práctica de la asignatura.

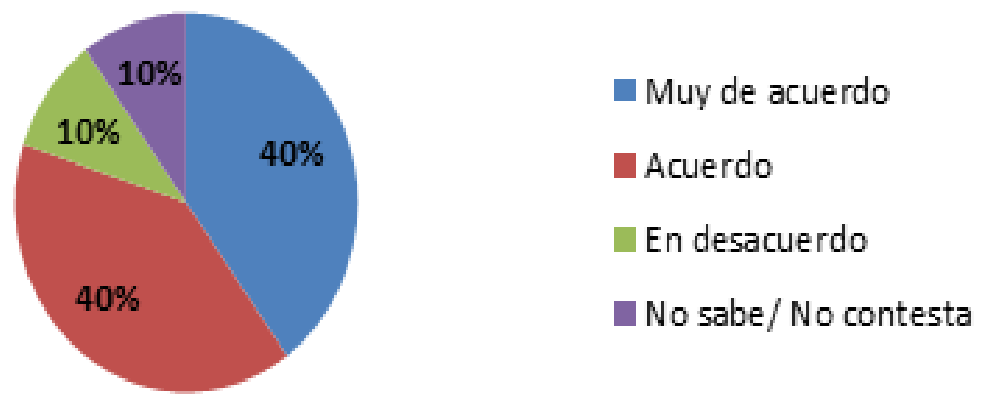

Figura 3.

Respuestas a la pregunta: "Creo que he aprendido más así que si hubiera realizado una práctica equivalente convencional”.

El 60\% les gustaría que el resto de prácticas de la asignatura se complementara de esta manera, es decir, relacionándolas con el desarrollo del Museo Geológico Virtual de la Universidad de León.

La Figura 4, muestra que el $90 \%$ de los estudiantes considera que la participación de los alumnos de las asignaturas relacionadas con el Museo Geológico Virtual de la Universidad de León es interesante para el desarrollo del museo.

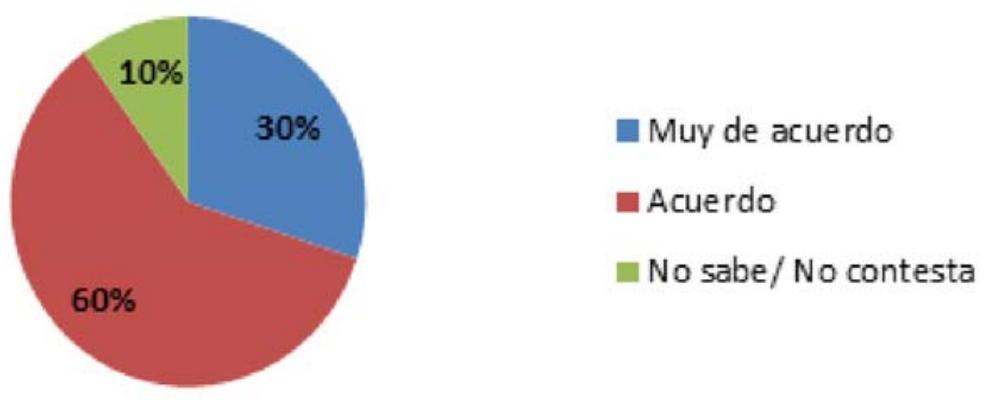

Figura 4.

Respuestas a la pregunta: ¿Considerarías que la participación de los alumnos de las asignaturas relacionadas con el Museo Geológico Virtual de la Universidad de León es interesante para el desarrollo del museo? 
Ante la pregunta sobre lo que les resultó más o menos interesante al realizar esta experiencia, se puede destacar que los alumnos entendieron que el aprendizaje del trabajo en grupo y la búsqueda de información, así como el mayor grado de implicación suponen grandes ventajas, mientras que el excesivo tiempo empleado y la escasez de medios adecuados aparecen como mayores dificultades (Figura 5)

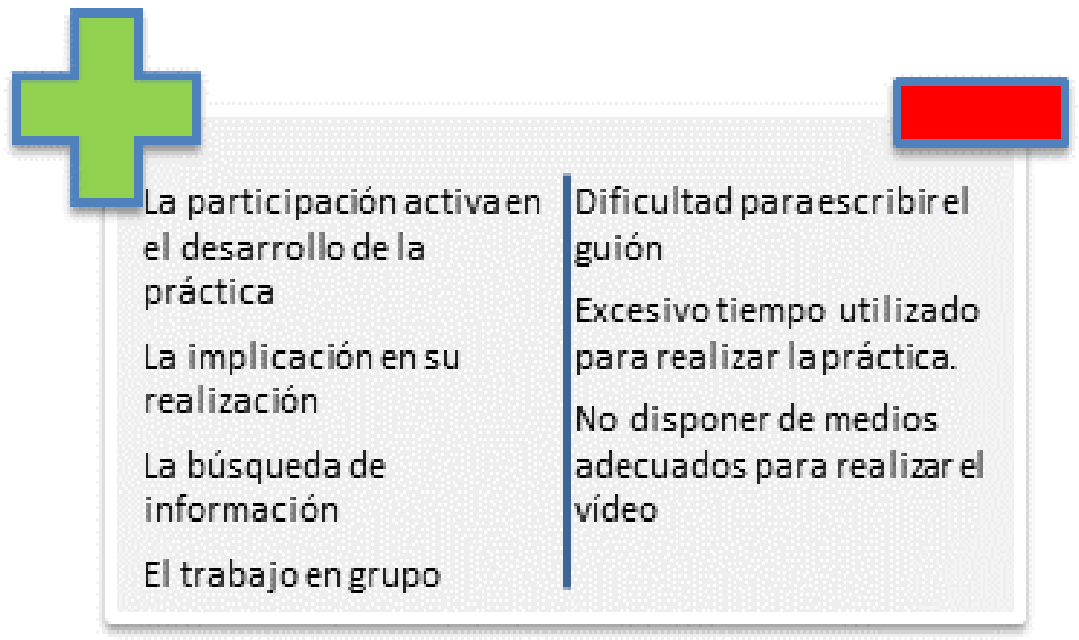

Figura 5.

Comentarios sobre la práctica realizada ante la pregunta:¿Qué es lo más positivo y lo más negativo del desarrollo de la práctica realizada?

Algunos encuestados realizaron sugerencias para futuras prácticas, donde animan a continuar realizando y ampliando experiencias de este tipo. También sugirieron la posibilidad de disponer de más ayuda técnica a la hora de realizar los vídeos, para mejorar su calidad.

Por lo que respecta a los resultados académicos de la "Prueba de reconocimiento y clasificación de minerales”, realizada por los alumnos tras la innovación docente (calificación media obtenida $=5.8$ puntos sobre 10), se puede decir que son similares, aunque ligeramente superiores a la media de los cursos académicos anteriores.

Dado el reducido número de alumnos que han participado en esta experiencia (10), los resultados no son concluyentes. Sin embargo, sirven como una primera aproximación para depurar futuras líneas de trabajo. Por ello, durante el presente curso y cursos posteriores se pretende ampliar el muestreo a alumnos de otras asignaturas de los Grados de Ingeniería Minera e Ingeniería de la Energía, con objeto de obtener una evaluación fiable de este y otros posibles proyectos de innovación ligados a la docencia de las disciplinas geológicas en grados de ingenierías 


\section{Referencias}

Alfonso, P., Font, J.M., Casas, J.M., Orche, E., \& Gómez-Fernández, F. (2009). Resumen de propuestas de la primera reunión de profesores de geología en ingeniería de minas, Andorra (Teruel). Segundo Congreso Internacional sobre Geología y Minería en la Ordenación del Territorio y en el Desarrollo. Utrillas, MC.27 305-308.

Fernández Caliani, J.C., de la Rosa, J.D., Requena, A., Casalvázquez, M.A., \& Galán, E. (2012). Potenciación del aprendizaje de la Mineralogía en un entorno didáctico virtual. Nuevos estándares en la innovación docente en Historia Natural. Actas del I Congreso Internacional de Innovación Docente Universitaria en Historia Natural, 43-49.

Sabbatini, M. (2004). Museos y Centros de Ciencia Virtuales. Complementación y Potenciación del Aprendizaje a través de Experimentos Virtuales. Tesis Doctoral. Universidad de Salamanca. 